\title{
$\$$ Research Square

\section{Noise Immune Dielectric Modulated Dual Trench Transparent Gate Engineered MOSFET as a Label Free Biosensor: Proposal and Investigation}

DIPANJAN SEN ( $\sim$ sendipanjan10@gmail.com )

Jadavpur University https://orcid.org/0000-0002-5781-4027

Arpan De

Jadavpur University

Bijoy Goswami

Jadavpur University

Sharmistha Shee

Jadavpur University

Subir Kumar Sarkar

Jadavpur University

\section{Research Article}

Keywords: Transparent Gate, Dual Trench Cavity, Inner Gate, Dielectric Modulation, Biosensor, Noise Assessment

Posted Date: May 28th, 2021

DOI: https://doi.org/10.21203/rs.3.rs-262352/v1

License: (c) (i) This work is licensed under a Creative Commons Attribution 4.0 International License. Read Full License

Version of Record: A version of this preprint was published at Journal of Computational Electronics on September 30th, 2021. See the published version at https://doi.org/10.1007/s10825-021-01780-x. 


\section{Abstract}

In this work, we have examined and proposed a dielectrically modulated biosensor based on the dual trench transparent gate engineered MOSFET (DM DT GE-MOSFET) for label-free detection of biomolecules with enhanced sensitivity and efficiency. Different sensing parameters such as the $\mathrm{I}_{\mathrm{ON}} / \mathrm{I}_{\mathrm{OFF}}$, threshold voltage shift have been evaluated to validate the sensing metric for the proposed device. Additionally, the $\mathrm{S}_{\mathrm{Vth}}\left(\mathrm{V}_{\text {th }}\right.$ Sensitivity) has been also analyzed by considering the charged (positive and negative) biomolecules. In addition to this, the RF sensing parameters such as the transconductance gain and cut-off frequency have been also taken into account to provide a better insight into the sensitivity analysis of the proposed device. Furthermore, the linearity, distortion and noise immunity of the device has been evaluated to check the overall performance of the biosensor at high frequency $(\mathrm{GHz})$. Moreover, the results indicate that, the proposed biosensor exhibits a $S_{V t h}$ of 0.68 for the positively charged biomolecules at a very low drain bias $(0.2 \mathrm{~V})$. Therefore, the proposed device can be used as an alternative to the conventional FET-based biosensors.

\section{Introduction}

Detection of nanoscale biomolecules and accurate analysis of those biomolecules is becoming inevitable day by day due to several new age findings in the field of microbiology. Identification of hundreds of proteins, such as DNA, biotin-streptavidin, S-protein and different kinds of bacteria and virus are crucial to understand any type of peculiar behavior inside a living cell. In addition to this, the pathological results related to COVID-19 shows an analogy among the previously reported SARS and MERS pandemic and the biopsy samples of the lung tissues clearly depicts a lesion in bilateral diffuse alveolar with cellular fibromyxoid exudates. Therefore, the importance of designing more efficient, highly sensitive and low cost biosensors have increased drastically.

Among all, the Field Effect Transistor (FET) based biosensors are of great research interest [1]. They provide several advantages among which CMOS compatibility, high scalability, label-free detection and low-cost production make them a promising candidate for future biosensor applications [2-3]. Significant usage in label free detection of charged bio-analytes has been seen for FET based biosensors [4-7]. The formation of a vertical nanogap with the biosensor has enabled the detection of charge free molecules as well [7-12]. The modulation of the coupling between gate and channel based on different charged/noncharged bio-analytes with varied range of permittivity is the working basis of dielectric modulated FET based biosensor [13]. To cite a few, APTES (K=3.57), Streptavidin ( $K=2.1)$, Biotin ( $K=2.63)$ [14], food proteins such as Gluten ( $K=5)$, Keratin ( $K=10)$, Zenin ( $K=7)$, Gelatin $(K=12)$ [15] have different permittivity but are non-charged in nature, whereas, among charged bio-analytes, amino acids such as glutamic acid, aspartic acid, arginine, lysine, histidine ( $\mathrm{K}=11-25)$ [16] are some examples. Moreover, the S-protein of the SARS-CoV-2 virus usually has glycoproteins in it and the dielectric constant of that protein lies in the range of 1-4 [17], similar to biotin or streptavidin. All these biomolecules have been successfully detected by previously reported FET based biosensors. With focus on dielectric modulated biosensing 
applications, split gate JL MOSFET [18], gate underlap DG MOSFET [19-20], surrounding gate MOSFET [21] have been reported.

In this paper, we have proposed a noise immune dual trench MOSFET (DM DT GE-MOSFET) based biosensor with split fashioned transparent (ITO) outer gate structure coupled with an inner gate. The architecture provides a better control over the channel profile through both exterior and interior gate arrangement. The inclusion of the inner gate enhances the device performance by forming an additional inversion layer. Improved drain current and decent threshold voltage shift makes the device suitable for low power biosensing applications. The biosensing capabilities of the device have been exhibited for various bio-analytes among which DNA, proteins etc. are noticeable ones. Tied-gate, symmetric arrangement with dielectric modulation forms the basis of working for our proposed structure. Cavity has been created on the top and bottom of the device with split-gates in between forming a trench like architecture. Furthermore, the device shows impressive results in terms of RF sensing metric and noise performance.

\section{Device Structure, Simulation Framework And Calibration Methodology}

The two dimensional schematic (top view) diagram and the possible fabrication steps of the proposed device has been shown in Fig. $1(a-b)$. Here, the proposed device has a dual trench split gate architecture with an additional "Inner" gate (IG) at the source side. The thickness for the outer gates on top and bottom is $2 \mathrm{~nm}$ and that of IG is $1 \mathrm{~nm}$, which is buried inside the silicon film. The length of the channel $\left(L_{C}\right)$ is $50 \mathrm{~nm}$, whereas the source length $\left(L_{S}\right)$ and drain length $\left(L_{D}\right)$ is 20 and $30 \mathrm{~nm}$ respectively. $A$ nanocavity has been embedded at the top and bottom of the device with intermediate positioning of control gates, which has a length of $15 \mathrm{~nm}$. Moreover, the maximum height of the biosensor cavity ( $T_{\text {CAVITY }}$ ) is $4 \mathrm{~nm}$. For immobilization of biomolecules within the cavity, a layer of oxide film is considered. The layer of oxide is formed by exposition of silicon surface to outer atmosphere and the thickness of $\mathrm{SiO} 2\left(\mathrm{~T}_{\mathrm{OX}}\right)$ to be grown is considered to be $1 \mathrm{~nm}$ for our analysis. Here, a $\mathrm{SiO}_{2}$ layer has been used, outlining the inner gate (IG) to avoid direct connection with silicon film. All the gates are given the same external bias for biosensing analysis. The length of the gates used for top and bottom parts are $20 \mathrm{~nm}$, whereas the inner gate length is $25 \mathrm{~nm}$. The source and drain regions are highly doped with $\mathrm{n}+$ dopants $\left(10^{19} \mathrm{~cm}^{-3}\right)$, while the channel is lightly doped p-type silicon, almost intrinsic in nature. In addition to this, ITO (indium doped tin oxide) has been used as the gate metal to enhance the on current of the proposed device with a workfunction of $4.7 \mathrm{eV}$. Also, a gate bias $\left(\mathrm{V}_{\mathrm{GS}}\right)$ of $0.7 \mathrm{~V}$ and drain bias $\left(\mathrm{V}_{\mathrm{DS}}\right)$ of $0.2 \mathrm{~V}$ has been considered throughout the analysis of the proposed device. Furthermore, Quantum Confinement issues are bound to crept into nanoscale devices but they do not exhibit significant effect in this case as the silicon film thickness is greater than $7 \mathrm{~nm}$. Hence, they are not included in our studies.

In this work, SILVACO ATLAS TCAD Tool [22] has been used as a potential candidate to extract the simulated results and figure of merits. Several physical models have been used, including the Shockley- 
Read-Hall model [22] for carrier recombination and generation. Lombardi (CVT) model has been considered to include the mobilities due to carrier scattering caused by the parallel and perpendicular electric fields applied on the device. Also, band gap narrowing or BGN model has been used here. FermiDirac distribution or statistics model has been used to increase the efficacy. Additionally, to include information related to the energy (temperature) of the charge carriers, the energy balance transport (EBT) model [22] has been added. In addition to this, the physical models used in the simulation set-up has been calibrated with the experimental results [23] shown in Fig. 2.

\section{Results And Discussion:}

\subsection{Electron Concentration Profile (lateral and vertical) of the proposed device:}

The electron Concentration profile of the device for different biomolecules are shown in Fig. 3 (a-b). It can be seen that as dielectric concentration of the biomolecules rises in the cavity, the electron concentration in the channel increases. This behavior can be attributed to the increase in electric field across the oxide layer due to higher permittivity, which causes more electrons to pop up from bulk and consequently increase the electron concentration in the channel (Fig. 3a). Moreover, Fig. 3b exhibits the same trend in the vertical direction as it can be seen along the channel. Here, the presence of the inner gate at the source/channel interface helps in creating sufficient amount of inversion layer throughout the channel region and thus reducing the on-resistance of the device. Therefore, the electron concentration profile of the proposed device depicts that, the inclusion of the extra inner gate at the source region forms a second inversion layer and thus it reduces the channel resistance drastically.

\subsection{Surface Potential characteristics of the proposed device:}

Figure 4 depicts the surface potential of the DM DT GE-MOSFET based biosensor against the variation of different biomolecules. Moreover, the immobilization of the biomolecules inside the nanocavity results into a change in the potential due to the change in the dielectric constant of the biomolecules. Furthermore, the shift in the surface potential can be used as a sensing metric to detect different biomolecules trapped inside the nanocavity region.

\subsection{Drain Current and $\mathrm{I}_{\mathrm{ON}} / \mathrm{I}_{\mathrm{OFF}}$ characteristics of the proposed device:}

3.4 Threshold Voltage and Threshold Voltage Sensitivity $\left(\mathrm{S}_{\mathrm{Vth}}\right)$ Profile of the proposed device:

Figure 6a shows the threshold voltage profile against the variation of different biomolecules. It is quite evident that, the threshold voltage of the device increases with the increase of the dielectric constant of 
the biomolecules. Here, the sensitivity of the DM DT GE-MOSFET based biosensor in the presence of different biomolecules in the nanocavity can be defined in terms of the shift in the threshold voltage as:

$$
\mathrm{S}_{\mathrm{V}_{\text {th }}}=\left|\frac{\mathrm{V}_{\mathrm{th}}(\text { air })-\mathrm{V}_{\mathrm{th}}(\text { bio })}{\mathrm{V}_{\mathrm{th}}(\text { air })}\right|
$$

where, the Vth (air) and Vth (bio) are the threshold voltages of the DM DT GE-MOSFET (measured with the help of constant current method at $I_{D}=10^{-7} \mathrm{~A} / \mu \mathrm{m}$ ) in the absence of biomolecules in the nanocavity (air-filled) and the presence of biomolecules in the nanocavity, respectively. The maximum change of threshold voltage is nearly $100 \mathrm{mV}$ at $\mathrm{K}=10$ (keratin filled) and the minimum change is $52 \mathrm{mV}$ at $\mathrm{K}=2$ (streptavidin filled) shown in Fig. 6b. Furthermore, the relative change in Vth shift values for keratin and streptavidin is $\sim 92.3 \%$, which indicates that the proposed device is highly sensitive in case of detecting specific biomolecules. Additionally, the absolute sensitivity profile shown in Fig. $6 \mathrm{~b}$ depicts that the proposed device is capable of detecting biomolecules at a very low supply voltage and thus it is suitable for low-power sensing application.

\subsection{Threshold Voltage Sensitivity $\left(\mathrm{S}_{\mathrm{Vth}}\right)$ for Charged Biomolecules (Positive and Negative):}

In addition, the DM MOSFET based biosensors are capable of providing high sensitivity against the charged biomolecules and the device type (n- or $p$ type MOSFET) needs to be changed to effectively capture the different polarity of charges depending on the ionized biomolecules 24 . These charges are generally localized at the surface of the gate dielectric layer and it modulates the surface potential of the channel region.

Figure $7(\mathrm{a}-\mathrm{b})$ depicts the effect of charged biomolecules on the sensitivity $\left(\mathrm{S}_{\mathrm{Vth}}\right)$ of DM DT GE-MOSFET based biosensor. Moreover, the positively charged biomolecules generally increase the surface potential, leading to an increased Vth shift and a consequent decrement in the threshold voltage. Similarly, the negatively charged biomolecules result in an increased threshold voltage and the Vth shift reduces. Therefore, the $\mathrm{S}_{\mathrm{Vth}}$ increases with an increased charge density of positively charged biomolecules at the $\mathrm{SiO}_{2}$ surface in the DM DT GE-MOSFET. The DM DT GE-MOSFET exhibits a significantly high sensitivity $\left(S_{V t h}\right)$ of 0.67 , when the nanocavity is filled with keratin $(K=10)$ at a positive charge density of $10^{12}$ $\mathrm{C} / \mathrm{cm}^{2}$ shown in Fig. $7 \mathrm{~b}$. However, the $\mathrm{S}_{\mathrm{Vth}}$ of the device degrades in the presence of negatively charged biomolecules inside the nanocavity at a $V_{D S}$ of $0.2 \mathrm{~V}$ as shown in Fig. $7 \mathrm{a}$.

\subsection{Sensitivity Assessment in terms of RF characteristics of the device:}


Here, the performance assessment of the proposed device is not only restricted to the investigation DC sensing metric, but the RF sensing metrics have been also analysed. The cut-off frequency $\left(f_{T}\right)$ and transconductance $\left(\mathrm{g}_{\mathrm{m}}\right)$ are the two essential parameters for investigating the RF performance of any biosensor device. Figure 8 a reveals an increment in $\mathrm{g}_{\mathrm{m}}$ against the increasing dielectric constant of the biomolecules and it exhibits the highest gain of $1.5 \times 10^{-3} \mathrm{~S} / \mu \mathrm{m}$ at a gate bias of $0.6 \mathrm{~V}$ in case of keratin filled $(K=10)$ cavity. Moreover, the proposed device exhibits a maximum sensitivity of 270 in terms of $g_{m}$ at $\mathrm{K}=10$ shown in Fig. $8 \mathrm{~b}$. Also, the relative change in sensitivity between $\mathrm{K}=2$ and 10 is nearly $267 \%$. In addition, the cut-off frequency or $\mathrm{f}_{\mathrm{T}}$ can be termed as the frequency at which the current gain is unity in the small-signal model of common source configuration. Thus, $\mathrm{f}_{\mathrm{T}}$ can be written as:

$$
f_{T}=\frac{g_{m}}{2 \Pi\left(C_{g d}+C_{g s}\right)}
$$

2

\subsection{Assessment of linearity and distortion profile of the proposed device:}

Furthermore, the linearity and intermodulation distortion (IMD) profiles are one of the pivotal parameters to analyse in case of a MOSFET based biosensor. To achieve higher speed and improved sensitivity in case of a FET based biosensor, less amount of distortion and improved linearity is required. Besides, nonlinearity introduces IMD and generates an undesired distorted signal, which results into the degradation of device performance. Additionally, the parameters used in analysing the biosensor performance in presence of noise are gm3 (higher order transconductance coefficient), third-order voltage intercept point (VIP3), third-order current intercept point (IIP3) and third-order IMD (IMD3) [25]. To achieve a linear behaviour, the higher order transconductance coefficient or gm3 must be as minimum as possible. As it limits the distortion level of the device and consequently fixes the dc bias point for optimal device performance. Figure 9a shows that gm3 has a peak value of $0.11 \mathrm{~A} / \mathrm{V}^{3}$ and overall value lies in the range $[-0.090 .11] \mathrm{A} / \mathrm{V}^{3}$. VIP3 signifies that extrapolated voltage and its peak value should be as high as possible to assert less distortion and high linearity [25]. Variation VIP3 with input $\mathrm{V}_{\mathrm{GS}}$ is shown in Fig. 9b. The peak of the VIP3 curve is about 1.4 V. Moreover, the point at which the input signal and third-order distortion signal amplitude converges is noted as IIP3.

Like VIP3, IIP3 should also have a high peak value. Figure 9c depicts the IIP3 profile and has a peak of $0.00125 \mathrm{~dB}$ (approx.) at a gate bias of $0.65 \mathrm{~V}$. Furthermore, another pivotal parameter to analyze the device reliability is IMD3, as it indicates the impact of nonlinearity which is a potent issue for linear amplifier. The intermodulation current at which the first- and third-order intermodulation harmonic currents are the same is represented by the IMD3. The IMD3 profile is shown in Fig. 9d, which depicts a peak value of $2.25 \mathrm{db}$. 
3.8 Noise Immunity Analysis of the proposed device:

Figure 10 (a-c) demonstrates the noise characteristics of the proposed device. In addition, the presence of any external element can sometimes lead to noise, which has a detrimental effect on the biosensor performance. Therefore, to investigate the noise immunity of DM DT GE-MOSFET based biosensor, noise parameters like the minimum noise figure, noise conductance and output source impedance etc. has to be analysed in depth [26]. Moreover, when the biomolecules are trapped and immobilized inside the nanocavity, all the noise FOMs seem to improve at high frequency. This observation can mostly be attributed to the transparent gate material (ITO) used in the DM DT GE-MOSFET architecture as the random motion of free charge carrier (electron) tend to reduce in such materials. Furthermore, in case of a transparent gate material, the electron temperature does not increase significantly with an increase in the concentration of charge carriers (electron). Thus, it results into improved noise immunity profile of the DM DT GE-MOSFET based biosensor in the presence of biomolecules. Here, the minimum noise figure for keratin ( $\mathrm{K}=10)$ filled cavity is $1.8 \mathrm{~dB}$ at a high frequency of $100 \mathrm{GHz}$ and thus it can be said that the proposed device is noise immune. Figure $10 \mathrm{c}$ illustrates another pivotal noise FOM, termed as the optimum source impedance, which is very close to zero at higher frequencies in case of air-filled cavity, but it gradually increases against the increasing dielectric constant of the biomolecule. Therefore, it indicates that the proposed device is capable of exhibiting very low noise in the presence of biomolecules and can be used as an alternative for biosensing applications.

\section{Conclusion}

In this work, the electrical characteristics of the proposed device have been analysed in terms of the threshold voltage shift, switching or $\mathrm{I}_{\mathrm{ON}} / \mathrm{I}_{\mathrm{OFF}}$ ratio, surface potential, $\mathrm{RF}$ sensitivity, linearity and noise FOMs. Here, the extracted results clearly exhibit that, the inclusion of ITO gate in the nanocavity enhanced the performance significantly of the DM DT GE-MOSFET based biosensor. Moreover, the proposed device exhibits a maximum $\mathrm{Vth}$ shift of nearly $100 \mathrm{mV}$ for $\mathrm{K}=10$. Furthermore, the DM DT GE-MOSFET based biosensor also depicts an impressive sensitivity profile in terms of RF sensing characteristics such as transconductance gain and cut-off frequency. It has been found that, the highest sensitivity in case of keratin $(K=10)$ also has a higher noise immunity at high frequency. Therefore, the DM DT GE-MOSFET based biosensor can be used as an emerging highly sensitive and noise immune device for detecting biomolecules based on dielectric modulation technique at low operating voltage.

\section{Declarations}

\section{Acknowledgement:}

The authors of this manuscript thankfully acknowledge the Department of ETCE, Jadavpur University for all the support.

\section{Conflict of Interest:}

The authors would like to state that they have no conflict of interest. 


\section{References}

1. Mukhopadhyay, S., Sen, D., Goswami, B., Sarkar, S.K.: "Performance Evaluation of Dielectrically Modulated Extended Gate Single cavity InGaAs/Si HTFET based Label-free Biosensor Considering Non-ideal Issues," in IEEE Sensors Journal, doi: 10.1109/JSEN.2020.3033576

2. Chen, X., Guo, Z., Yang, G.-M., Li, J., Li, M.-Q., Liu, J.-H., Huang, X.-J., “Electrical nanogap devices for biosensing," Mater. Today, vol. 13, no. 11, pp. 28-41, Nov. 2010

3. Narang, R., Reddy, K.V.S., Saxena, M., Gupta, R.S., Gupta, M., Dielectric-Modulated, "A., Tunnel-FETBased Biosensor for Label-Free Detection: Analytical Modeling Study and Sensitivity Analysis," in IEEE Transactions on Electron Devices, vol. 59, no. 10, pp. 2809-2817, Oct. 2012, doi:

10.1109/TED.2012.2208115

4. Bergveld, P.: The development and application of FET-based biosensors. Biosensors 2(1), 15-33 (1986)

5. Wenga, G., Jacques, E., Salaun, A.-C., et al.: Step-gate polysilicon nanowires field effect transistor compatible with CMOS technology for label-free DNA biosensor. Biosensors Bioelectron. 40(1), 141146 (Feb. 2013)

6. Guan, W., Duan, X., Reed, M.A.: Highly specific and sensitive nonenzymatic determination of uric acid in serum and urine by extended gate field effect transistor sensors. Biosensors Bioelectron 51, 225231 (Jan. 2014)

7. Kanungo, S., Chattopadhyay, S., Gupta, P.S., Rahaman, H.: "Comparative Performance Analysis of the Dielectrically Modulated Full- Gate and Short-Gate Tunnel FET-Based Biosensors". IEEE Trans. Electron Devices. 62(3), 994-1001 (March 2015). doi:10.1109/TED.2015.2390774

8. Im, H., Huang, X.J., Gu, B., et al.: A dielectric-modulated fieldeffect transistor for biosensing. Nature Nanotechnol. 2(7), 430-434 (2007)

9. Kim, C.H., Jung, C., Lee, K.B., et al., "Label-free DNA detection with a nanogap embedded complementary metal oxide semiconductor," Nanotechnology, vol. 22, no. 13, pp. 135502-1-135502-5, Apr. 2011

10. Gu, B., Park, T.J., Ahn, J.H., et al., "Nanogap field-effect transistor biosensors for electrical detection of avian influenza," Small, vol. 5, no. 21, pp. 2407-2412, Nov. 2009

11. Kim, C.H., Jung, C., Park, H.G., et al., "Novel dielectric modulated field-effect transistor for label-free DNA detection," Biochip J., vol. 2, no. 2, pp. 127-134, Jun. 2008

12. Choi, J.M., Han, J.W., Choi, S.J., et al., "Analytical modeling of a nanogap-embedded FET for application as a biosensor," IEEE

13. Im, H., Huang, X.-J., Gu, B., Choi, Y.-K.: A dielectric-modulated fieldeffect transistor for biosensing. Nat. Nanotechnol. 2(7), 430 (Jul. 2007), ",, , , - 434

14. Ajay, M., Gupta, R., Narang, Saxena, M., "Analysis of GaSb-InAs gate all around (GAA) p-i-n tunnel FET (TFET) for application as a biosensor," in Proc. IEEE Int. Nanoelectron. Conf. (INEC), May: 2016, pp. 1-2 
15. Bibi, F., et al.: "A Review: Origins of the Dielectric Properties of Proteins and Potential Development as Bio-Sensors." Sensors (Basel, Switzerland) vol. 16,8 1232. 4 Aug. 2016, doi:10.3390/s16081232

16. Goswami, R., Bhowmick, B., "Comparative analyses of circular gate TFET and heterojunction TFET for dielectric-modulated label free biosensing," in IEEE Sensors Journal., vol. 19, no. 21, pp. 96009609, Nov. 2019

17. Kemper Talley, C., Ng, M., Shoppell, Petras Kundrotas and Emil Alexov "On the electrostatic component of protein-protein binding free energy", PMC Biophysics, vol. 1, no. 2 Mar.2008. doi: 10.1186/1757-5036-1-2

18. Ajay, R., Narang, M., Saxena, Gupta, M.: Modeling of gate underlap junctionless double gate MOSFET as bio-sensor. Mater. Sci. Semicond. Process. 71, 240-251 (2017)

19. Chanda, M., Das, R., Kundu, A., Sarkar, C.K.: Analytical modeling of label free biosensor using charge plasma based gate underlap dielectric modulated MOSFET. Superlattices Microstruct. 104, 451-460 (2017)

20. Chakraborty, A., Sarkar, A.: Analytical modeling and sensitivity analysis of dielectric-modulated junctionless gate stack surrounding gate MOSFET (JLGSSRG) for application as biosensor. J. Comput. Electron. 16(3), 556-567 (2017)

21. Ajay, R., Narang, M., Saxena, Gupta, M.: Modeling and Simulation Investigation of Sensitivity of Symmetric Split Gate Junctionless FET for Biosensing Application. IEEE Sens. J. 17(15), 4853-4861 (2017)

22. ATLAS Device Simulation Software, Silvaco Int., Santa Clara, CA, USA, 2014

23. Appenzeller, J., Martel, R., Avouris, P., Knoch, J., Scholvin, J., del Alamo, J.A., Rice, P., Solomon, P.: Sub40 nm SOI V-groove n-MOSFETs. IEEE Electron Device Lett. 23(2), 100-102 (2002)

24. Kannan, N., Kumar, M.J., "Dielectric-Modulated Impact-Ionization MOS Transistor as a Label-Free Biosensor," IEEE Electron Dev. Lett., vol. 34, no. 12, pp. 1575-1577, Dec. 2013

25. Bhattacharyya, A., Chanda, M., De, D.: "Analysis of Noise-Immune Dopingless Heterojunction BioTFET Considering Partial Hybridization Issue". IEEE Trans. Nanotechnol. 19, 769-777 (2020). doi:10.1109/TNANO.2020.3033966

26. Kumar, A., Tripathi, M.M., Chaujar, R.: Ultralow-power dielectric-modulated nanogap-embedded sub20-nm TGRC-MOSFET for biosensing applications. J. Comput. Electron. 17, 1807-1815 (2018). https://doi.org/10.1007/s10825-018-1237-2

\section{Figures}




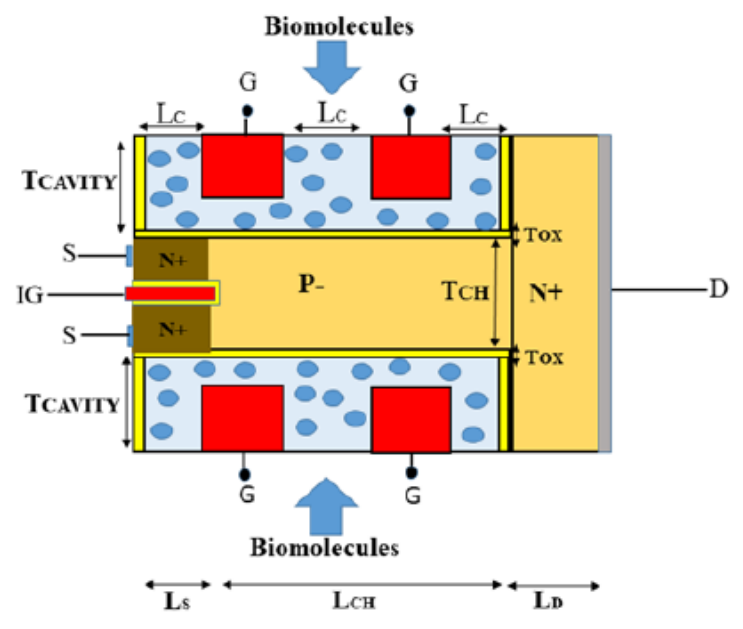

(a)

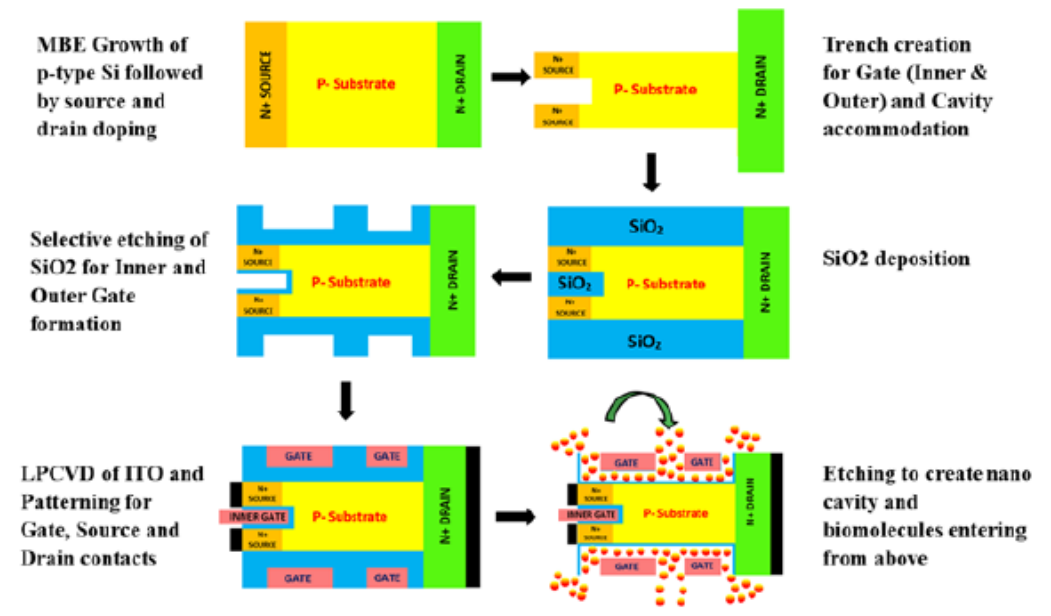

(b)

\section{Figure 1}

(a) 2D schematic (top view) representation and (b) possible fabrication steps of dual trench transparent gate engineered MOSFET (DM DT GE-MOSFET) based biosensor

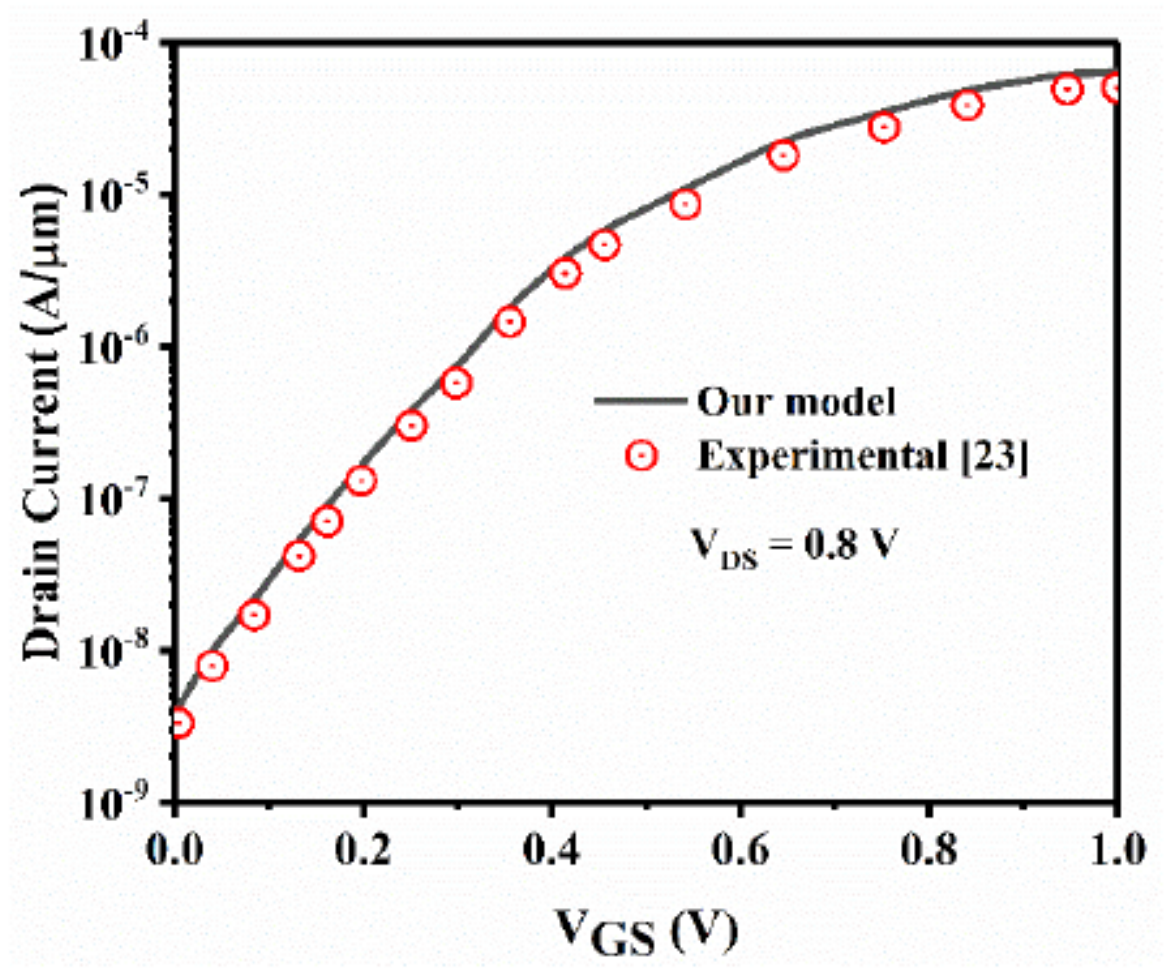

Figure 2

Calibration of simulation models with experimental data reported in [23] 


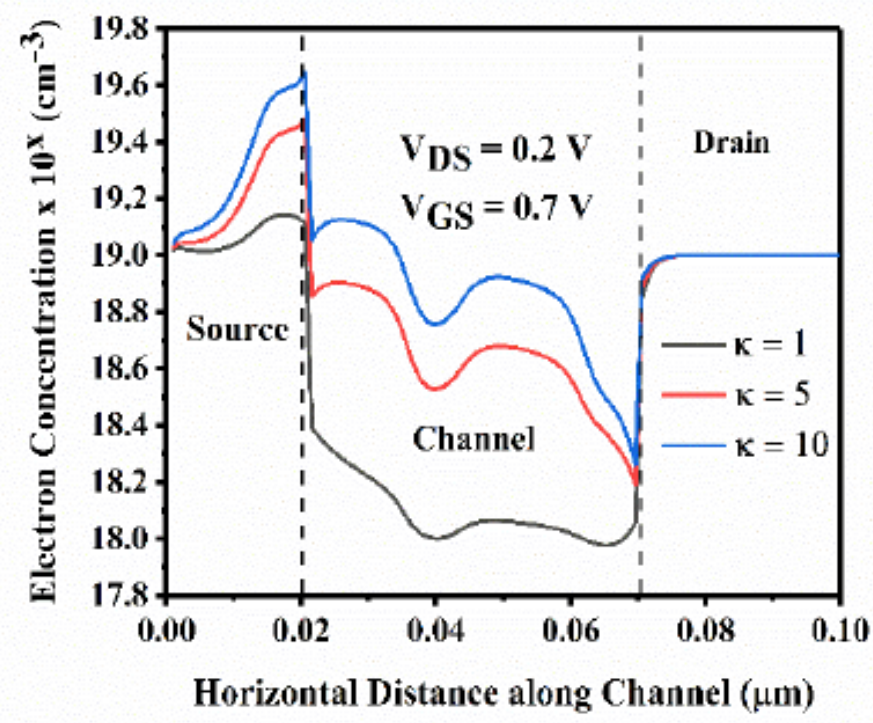

(a)

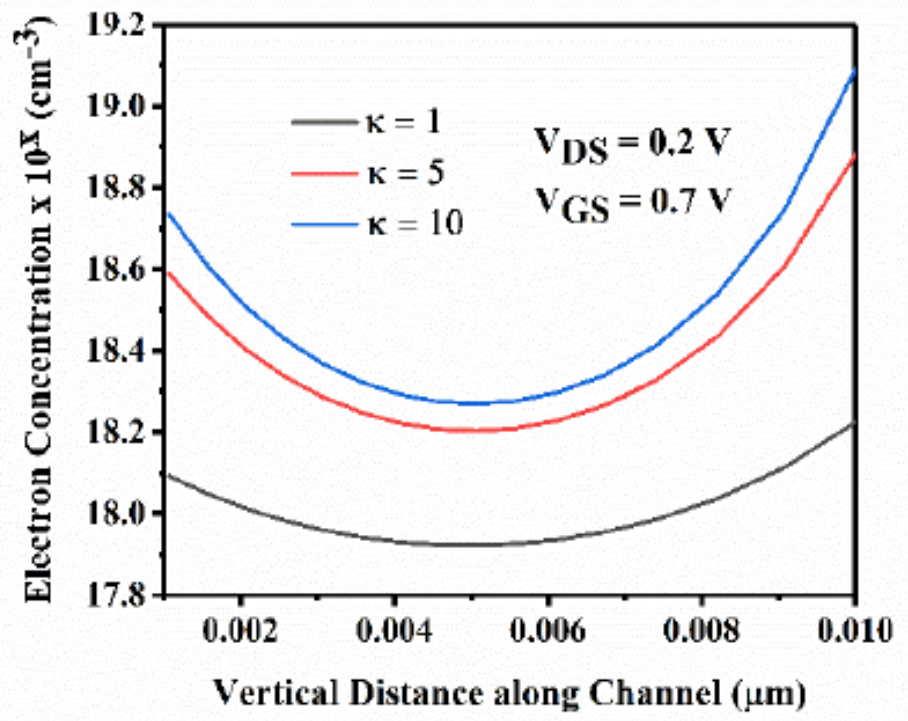

(b)

Figure 3

Electron Concentration profile of DM DT GE-MOSFET based biosensor (a) lateral direction (b) vertical direction

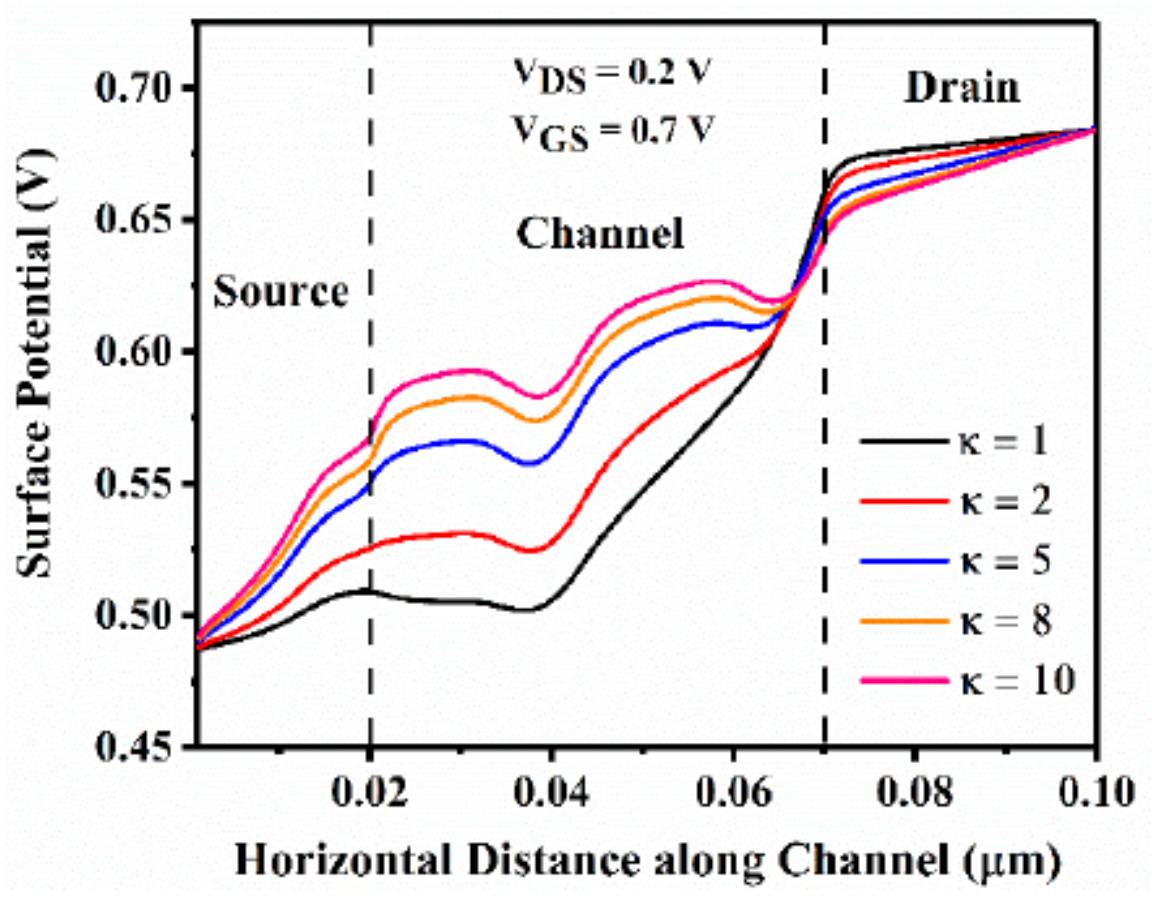

Figure 4

Surface Potential profile of DM DT GE-MOSFET based biosensor 


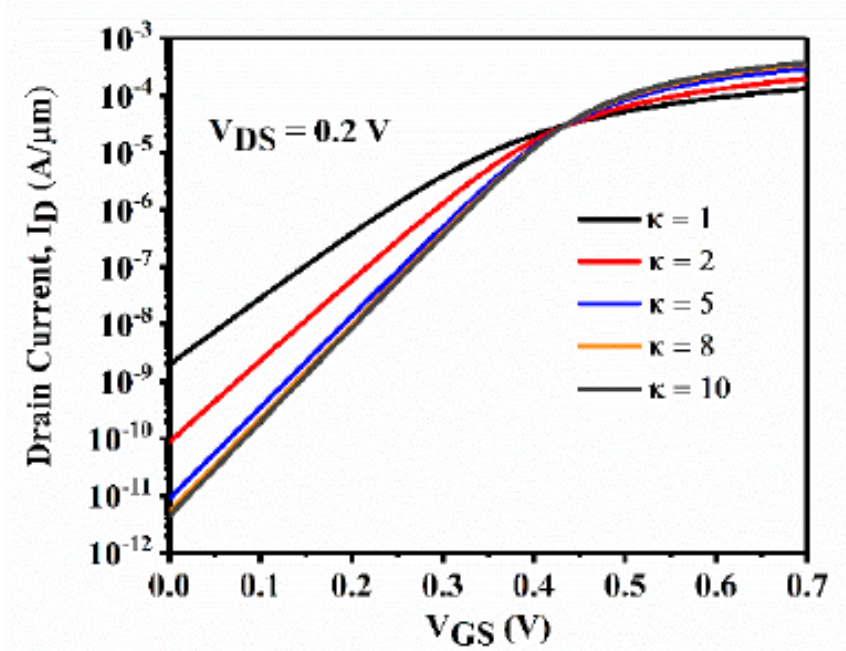

(a)

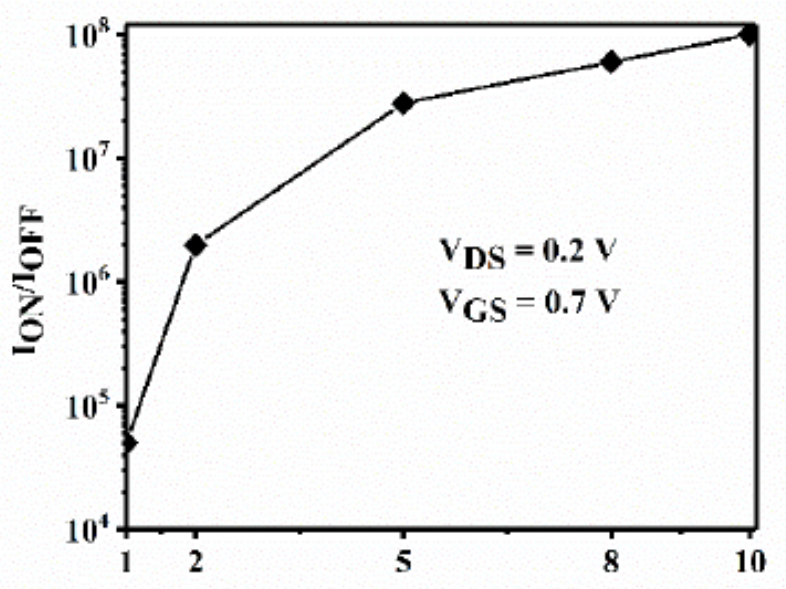

Dielectric Constant of Biomolecules ( $\kappa$ )

(b)

Figure 5

(a) Drain Current vs Applied Gate bias (b) Ion/loff ratio for different permittivity of wide range of biomolecules.

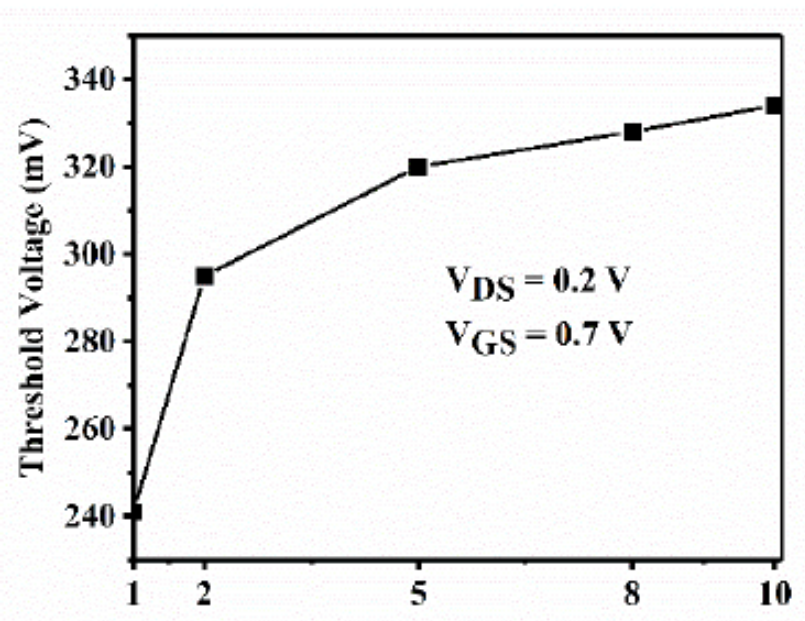

Dielectric Constant of Biomolecules ( $\kappa$ )

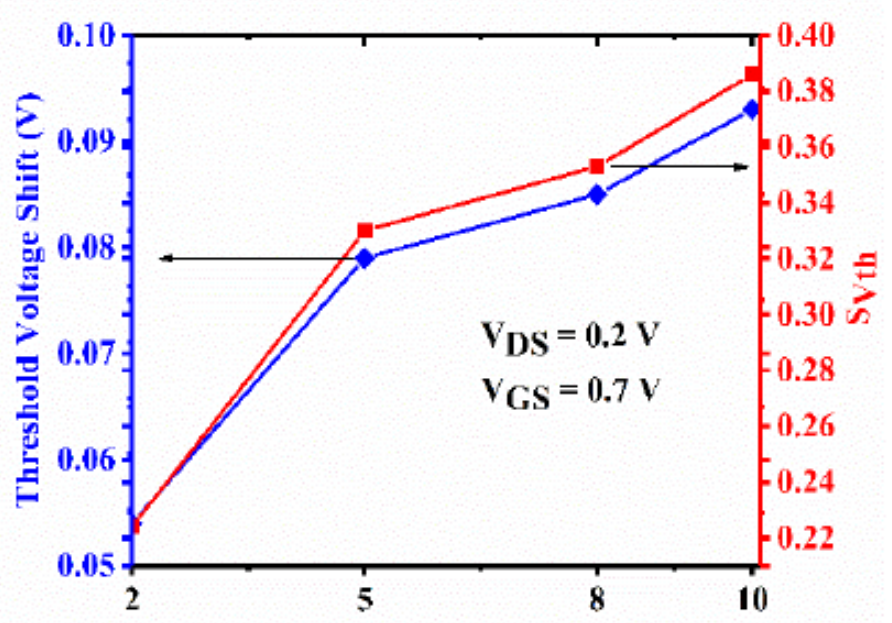

Dielectric Constant of Biomolecules ( $\kappa$ )

(a)

(b)

Figure 6

(a) Threshold Voltage and (b) Threshold Voltage Sensitivity (SVth) characteristics of DM DT GE-MOSFET based biosensor for neutral biomolecules 


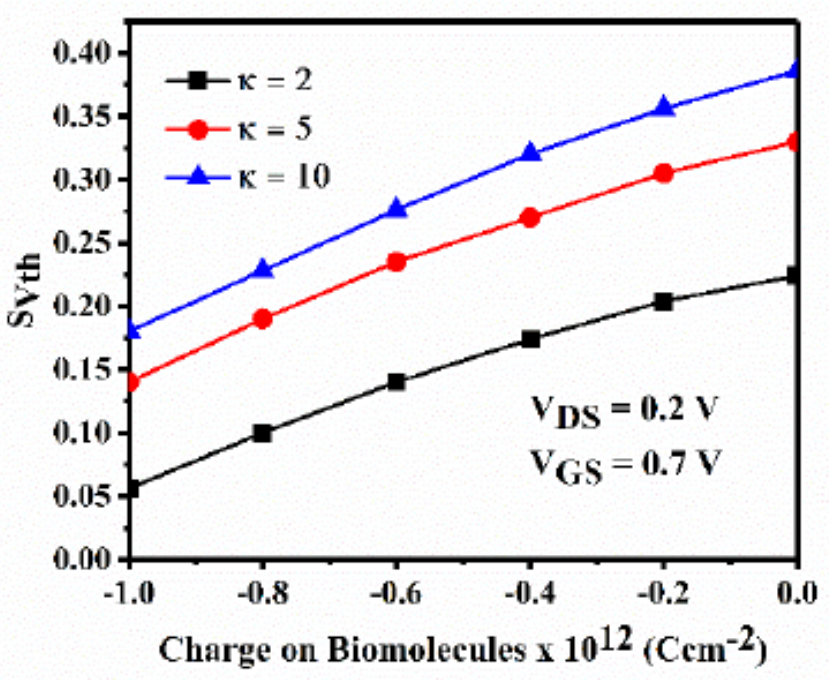

(a)

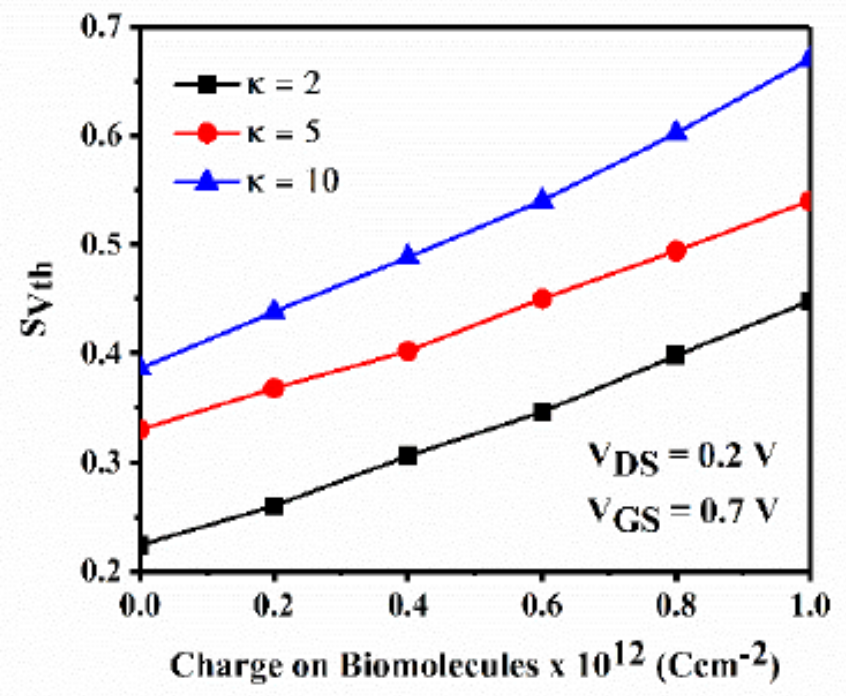

(b)

Figure 7

Sensitivities in terms of threshold voltage shift for (a) Negative Charged (b) Positive Charged bio-analytes 


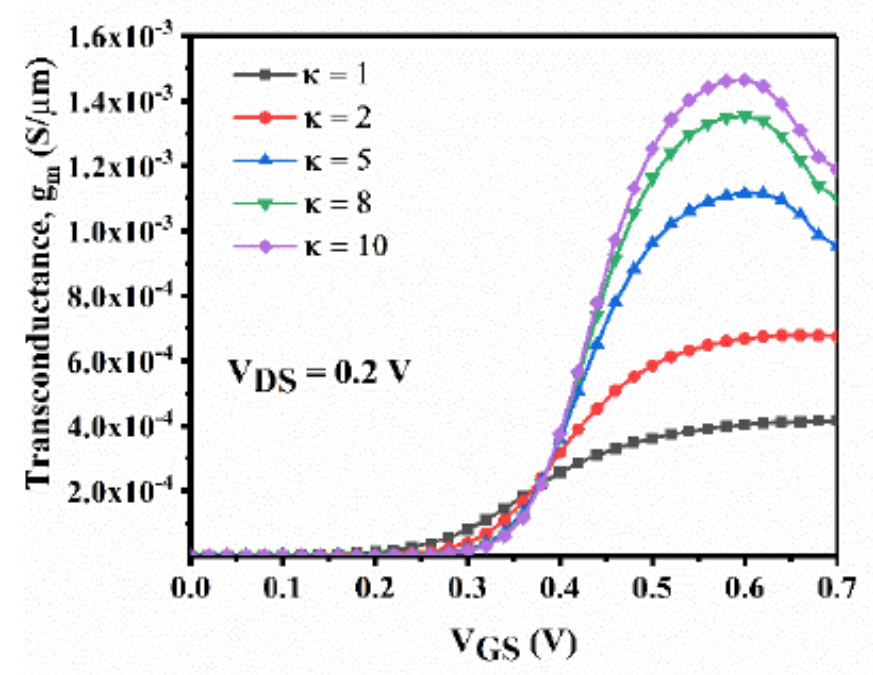

(a)

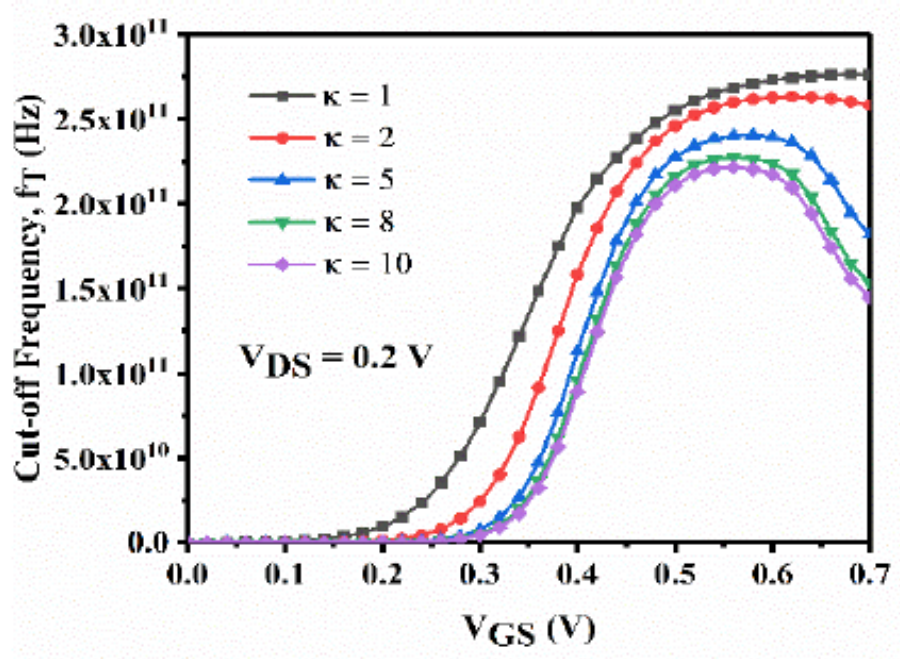

(c)

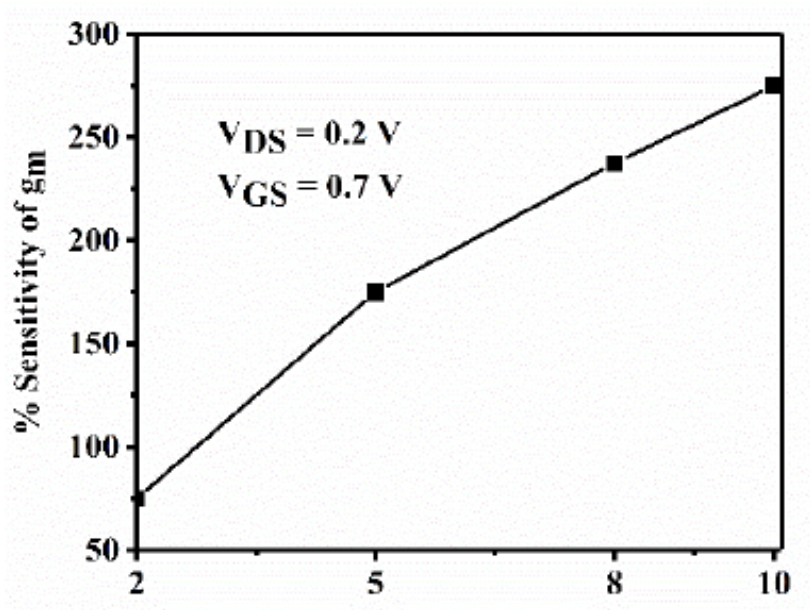

Dielectric Constant of Biomolecules (к)

(b)

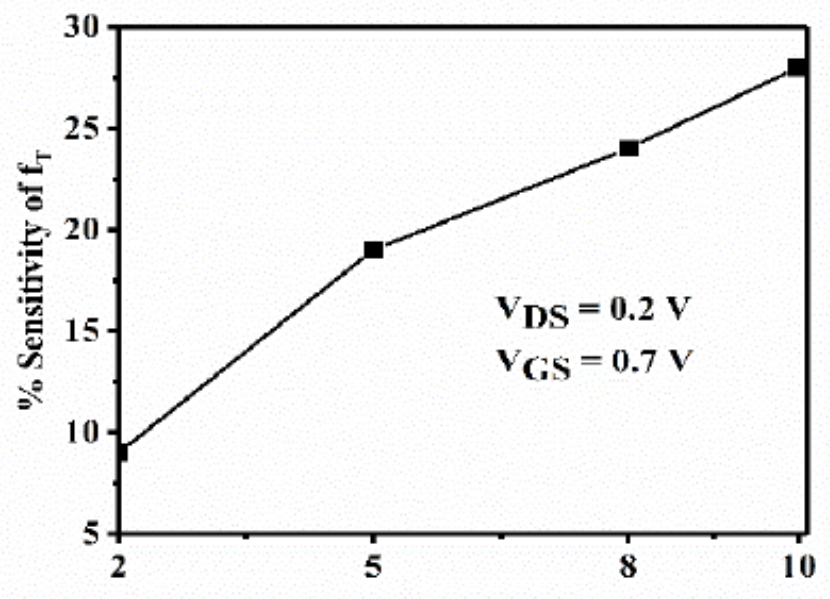

Dielectric Constant of Biomolecules (к)

(d)

Figure 8

(a) gm, (b) percentage sensitivity of gm, (c) fT and (d) percentage sensitivity of fT of the proposed device for different biomolecules 


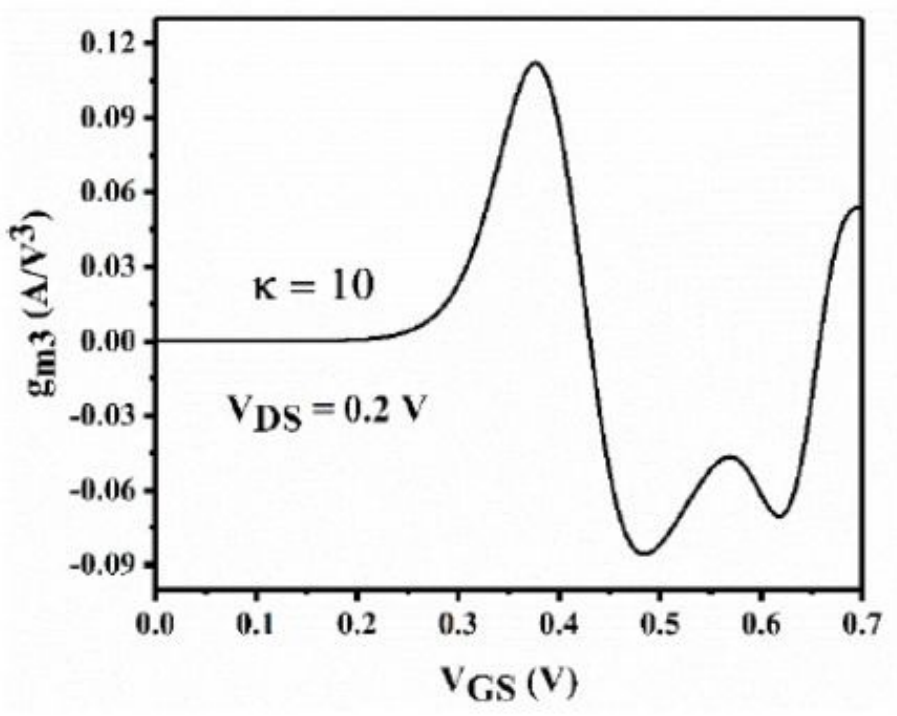

(a)

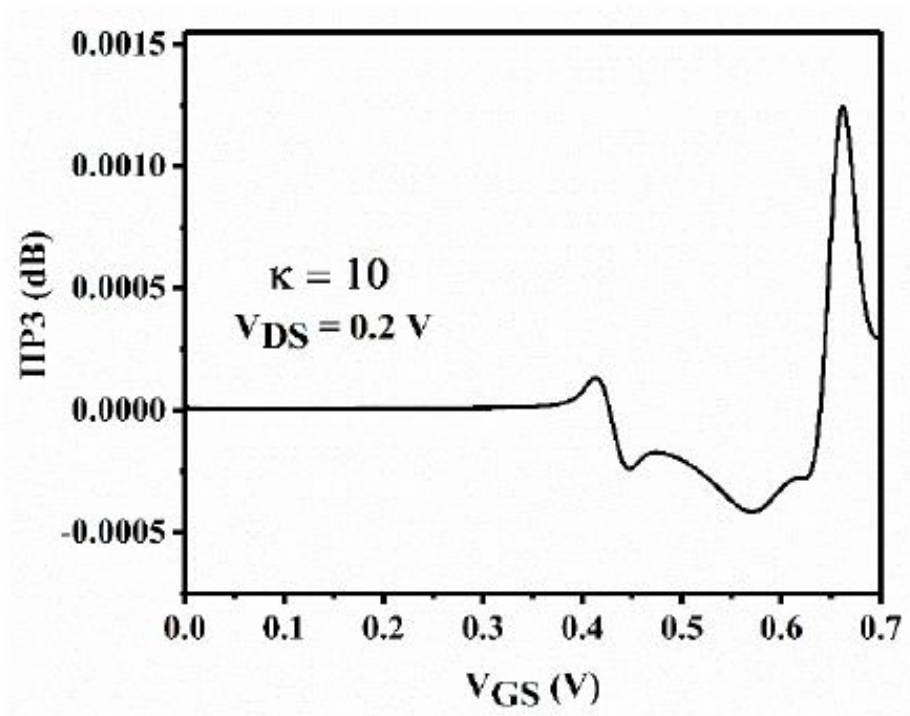

(c)

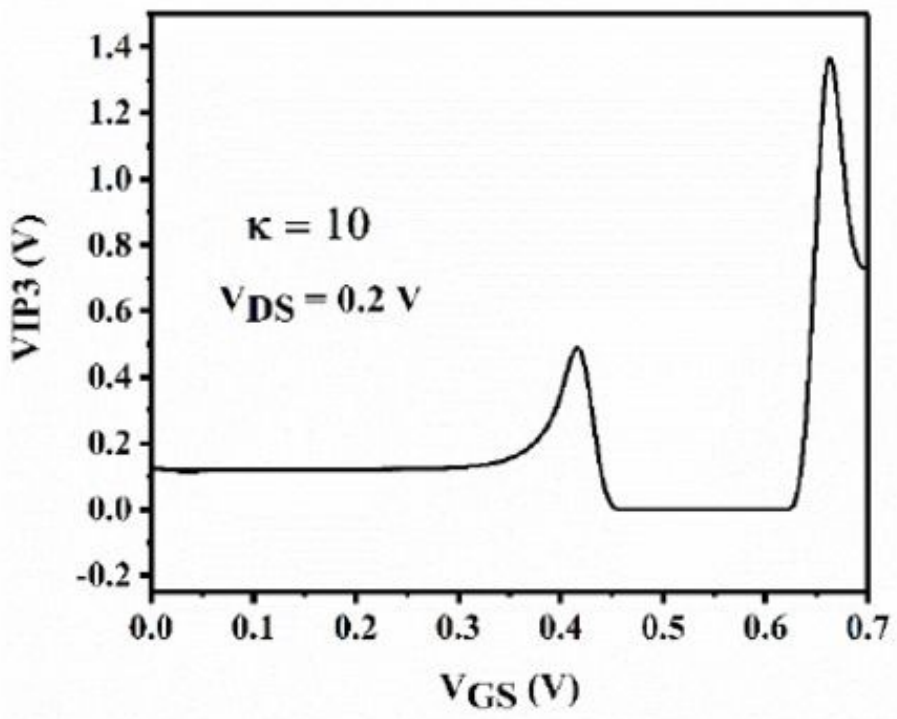

(b)

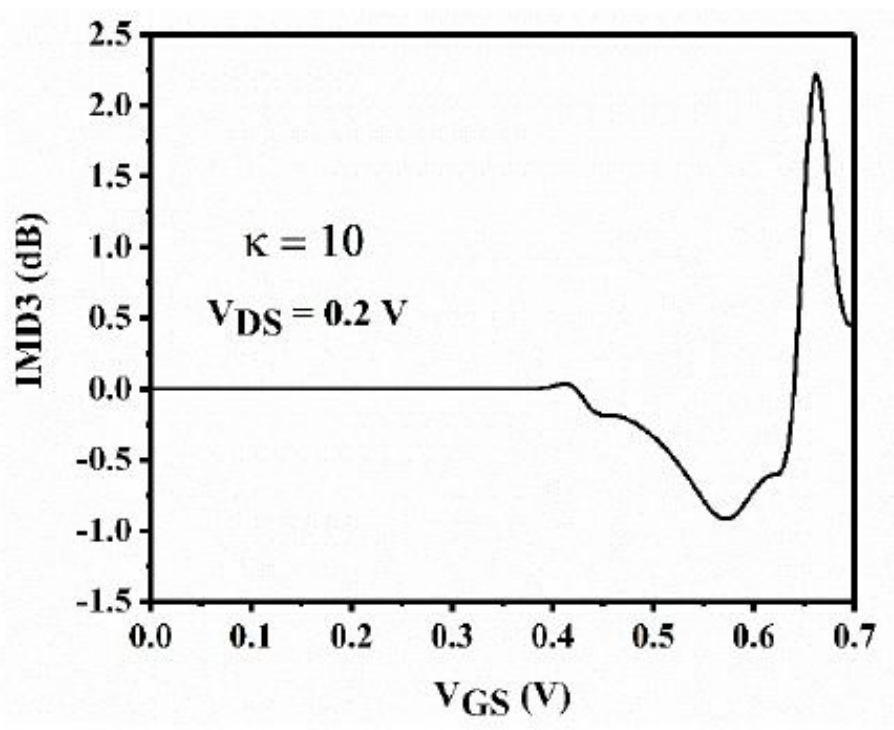

(d)

Figure 9

(a) gm3, (b) VIP3, (c) IIP3 and (d) IMD3 profile of proposed device for keratin $(k=10)$ at VDS $=0.2 \mathrm{~V}$ 


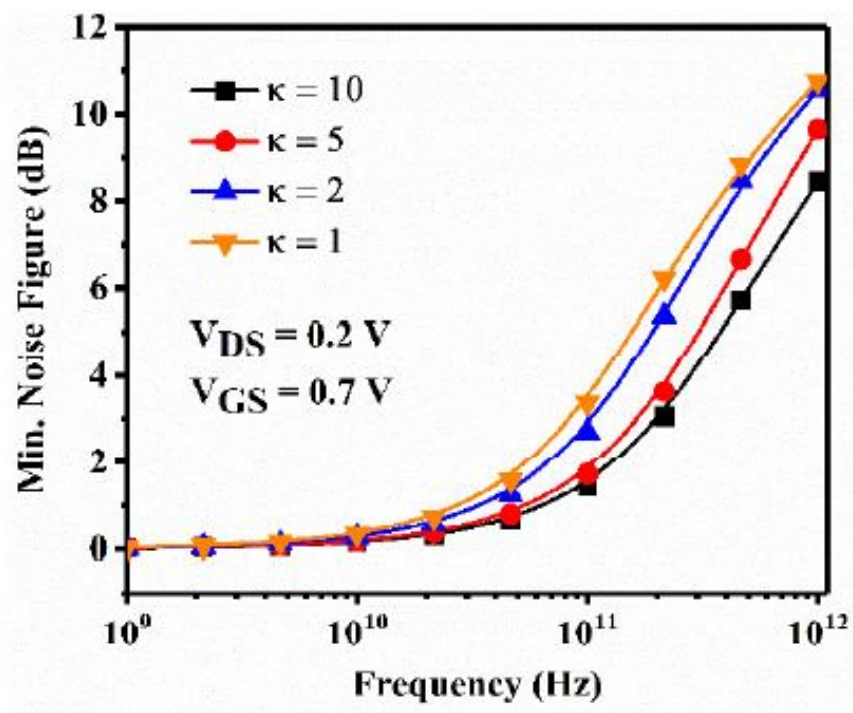

(a)

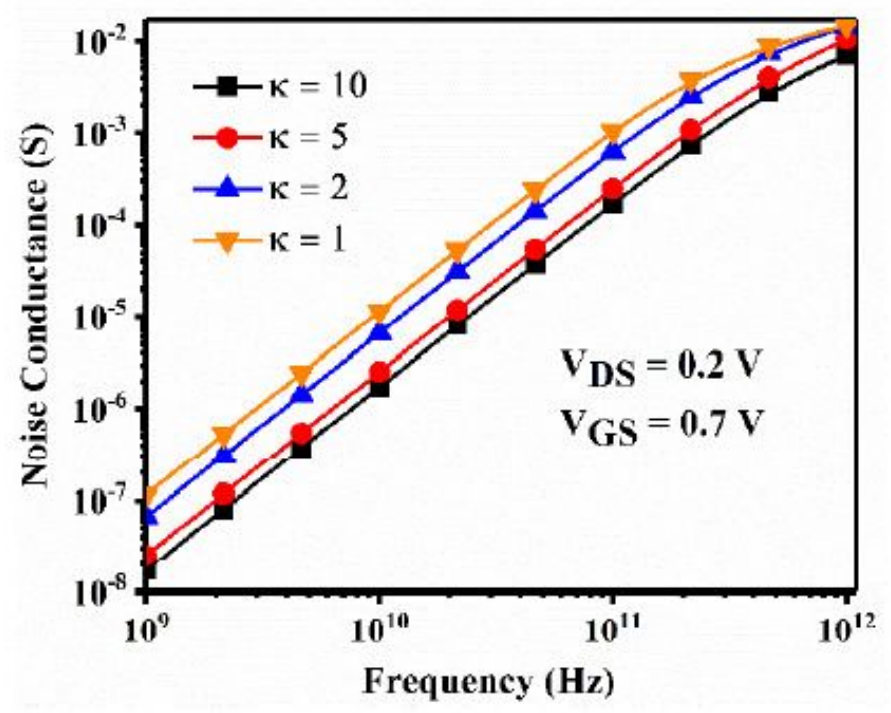

(b)

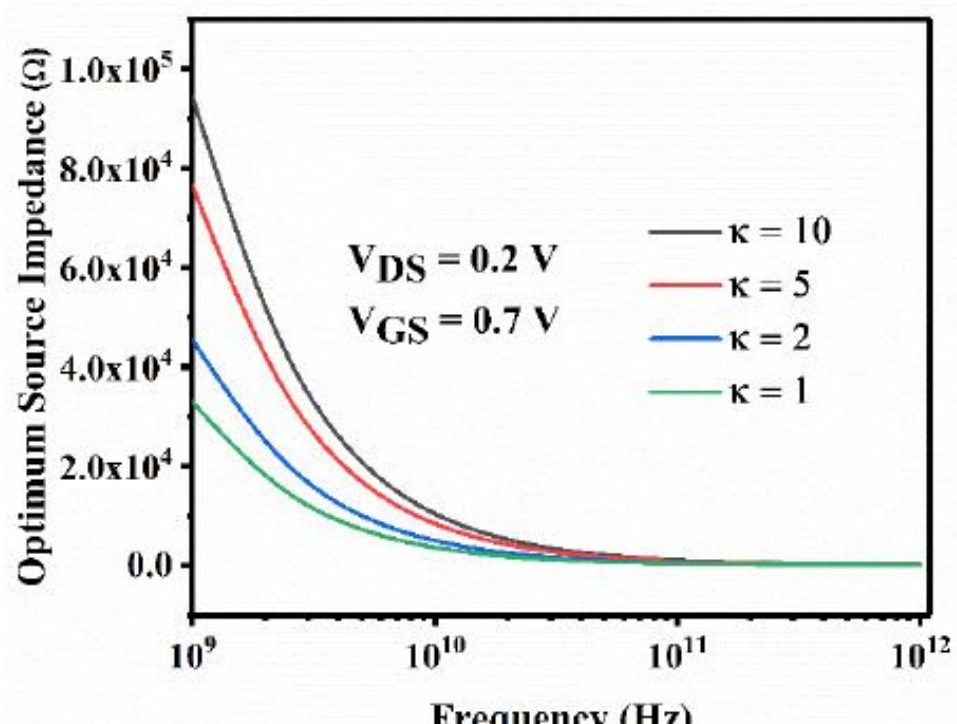

Frequency $(\mathrm{Hz})$

(c)

Figure 10

(a) Minimum Noise Figure, (b) Noise Conductance and (c) Optimum Source 